\title{
Can bone turnover markers help to define the duration of bisphosphonate drug holidays?
}

Louise Statham ${ }^{1,2}$, Sharon Abdy ${ }^{1}$, Terry Aspray ${ }^{1,3}$

${ }^{1}$ The Newcastle upon Tyne Hospitals NHS Foundation Trust, Newcastle upon Tyne, NE7 7DN, UK

2 University of Sunderland, Dept. of Pharmacy, Health \& Well-being, Sunderland, SR1 3SD, UK

${ }^{3}$ Newcastle University, Institute of Cellular Medicine, Newcastle upon Tyne, NE2 4HH, UK

University of

Sunderland

\section{Background}

Good evidence for 5 years bisphosphonate (BP) treatment ${ }^{1,2}$ but beyond this less clear

- $\quad$ BP long half-life; stopping treatment $\rightarrow$ wears off gradually ${ }^{2}$

Potential for harm

- $\quad$ Atypical fractures, osteonecrosis of the jaw (ONJ)

- $\quad$ Rare occurrence, $\uparrow$ risk with increasing duration ${ }^{3}$

BPs impair Bone Turnover; CTX bone turnover marker (bone resorption)

- $\quad$ Start BP $\rightarrow \downarrow C T X$

- $\quad$ Stop BP \& CTX rises $^{2}$
Drug holidays increasingly common - stop BP for period of time Local practice since late 2012

- Review BP after 5 yrs

- Drug holiday

- Routine monitoring CTX at baseline, 4 months and 12 months

Our aim was to analyse changes in CTX on stopping long term bisphosphonate treatment to guide clinical decision-making on the duration of treatment cessation

\section{Methods}

- Patients on BP drug holiday via outpatient Bone Clinic identified from monitoring records

- Data extracted; patient age, sex, serum CTX levels 0, 4, 12 months, bisphosphonate and duration of use

- Excluded if baseline (0m) CTX $\geq 0.51 \mathrm{ug} / \mathrm{L}$ (higher fracture risk)

- Data analysis using Stata Statistics software.

- Offset of action defined as

- a rise by the Least Significant Change $\left(\mathrm{LSC}=33 \%^{*}\right)$ in CTX and CTX above the pre-menopausal mean $(0.19 \mathrm{ug} / \mathrm{L})$

$* L S C=2.33 \times V\left(C V a^{2}\right)+\left(C V i^{2}\right): C V a$ is analytical coefficient of variation, $C V i$ is intra-individual $C V$
Figure 1: All patient characteristics

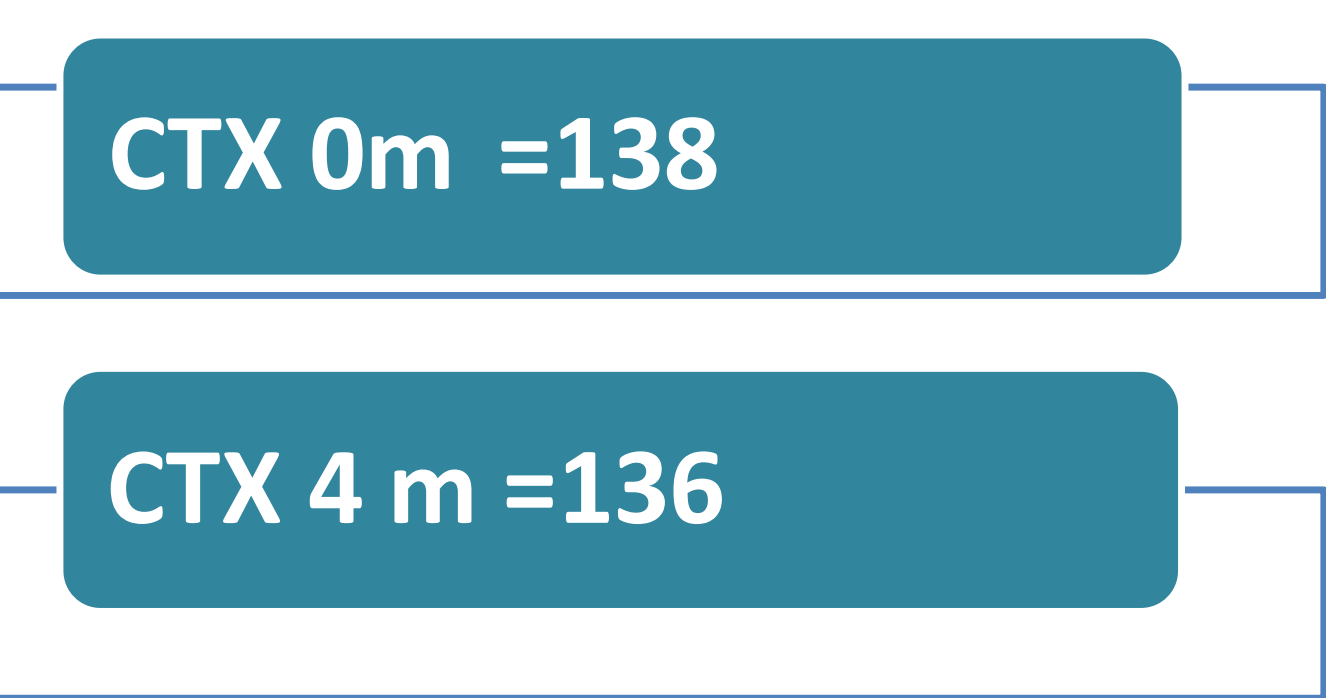

CTX $12 m=100$

Total Patients $=158$

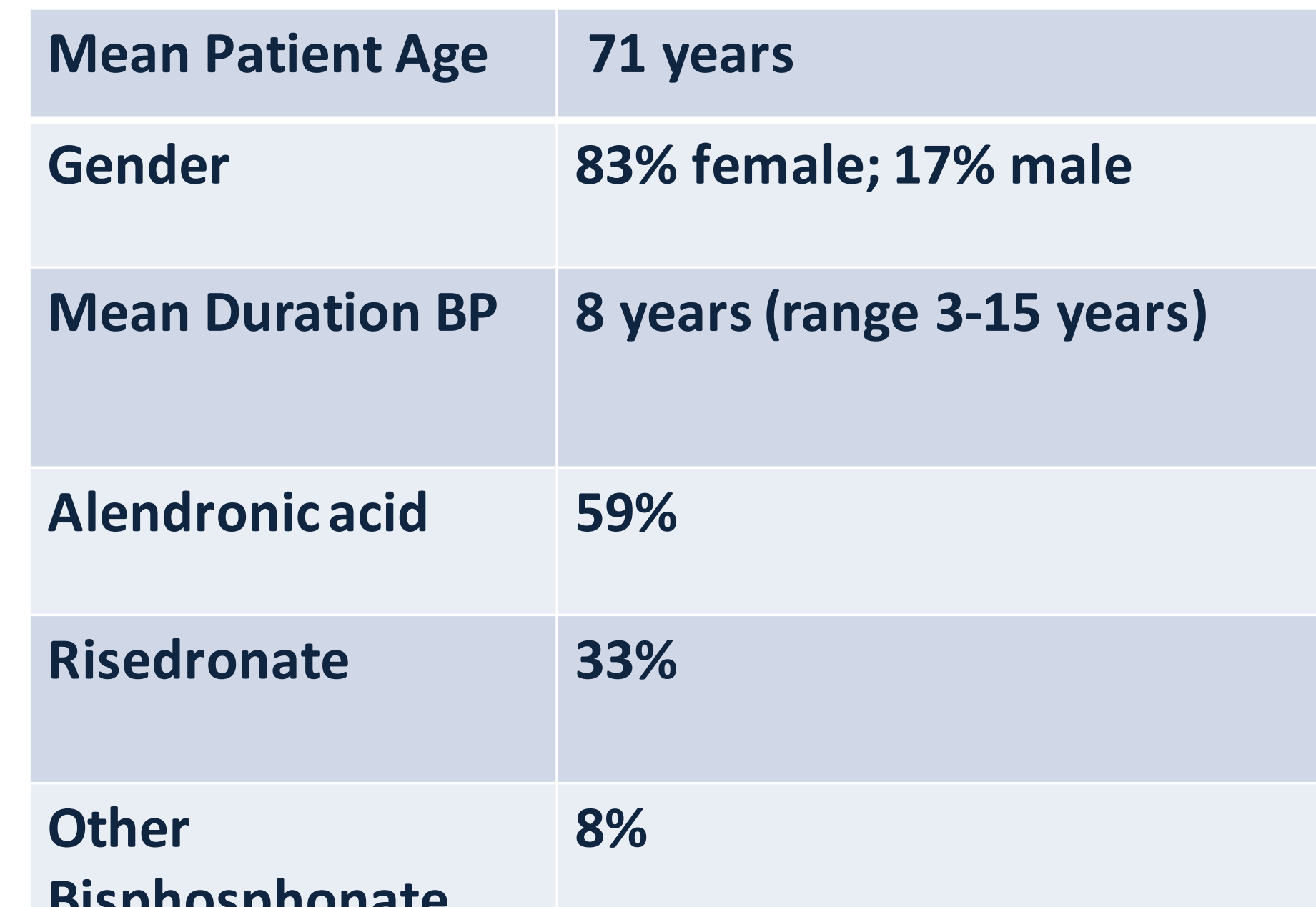

Bisphosphonate

\section{Results}

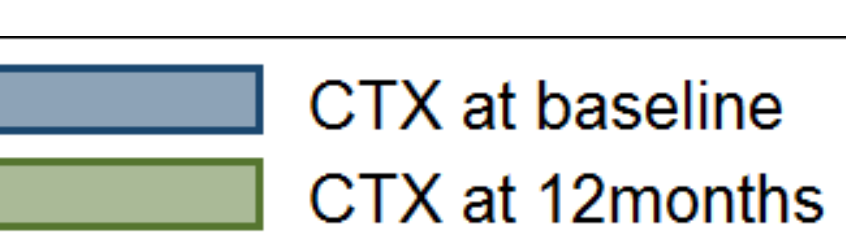

igure 2: CTX at 0, 4, 12 months all patients ( $<0.51$ at baseline)

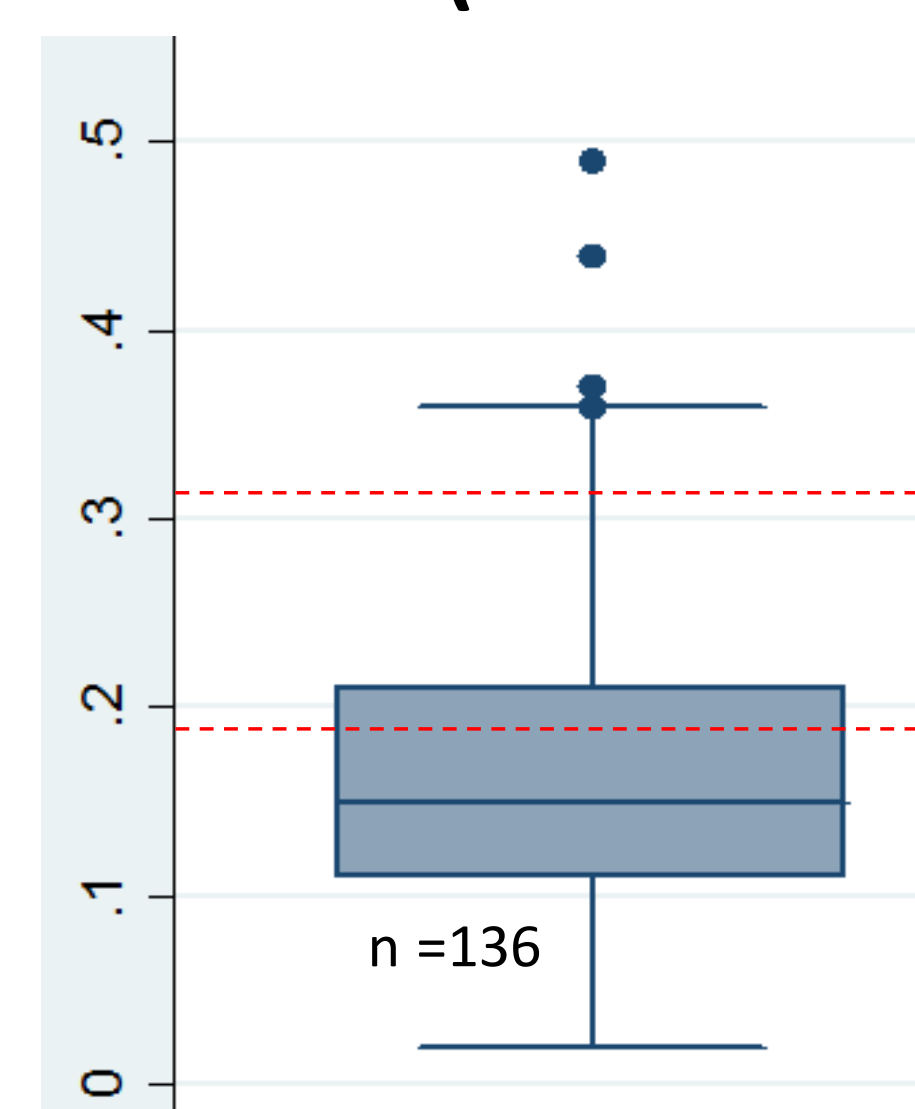

Delta CTX

Median [IQR] in ug/L Mean (SD) in ug/L

Significant rise (>LSC)

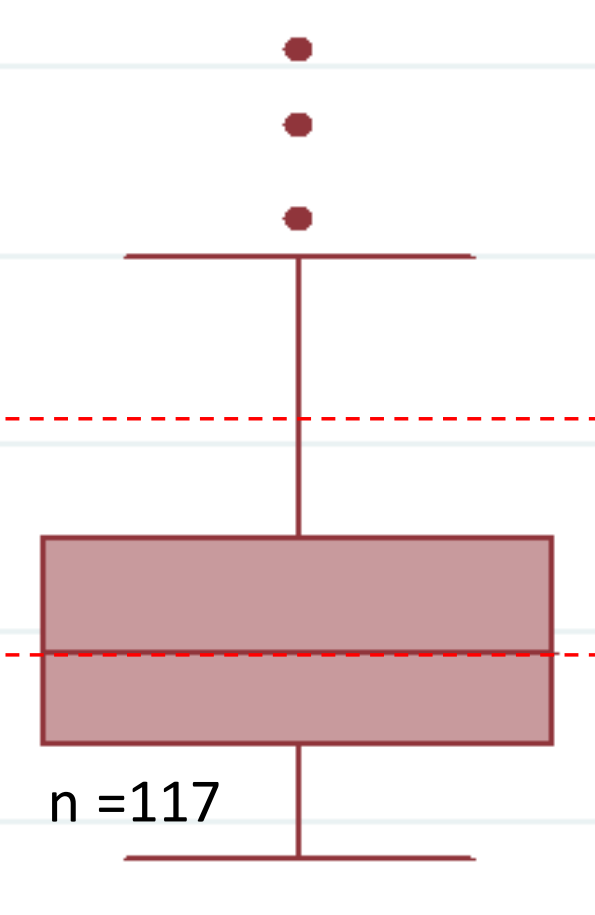

$+0.04[0.01-0.08]$ $+0.04(0.06)$ $+42 \%$

$47 \%$

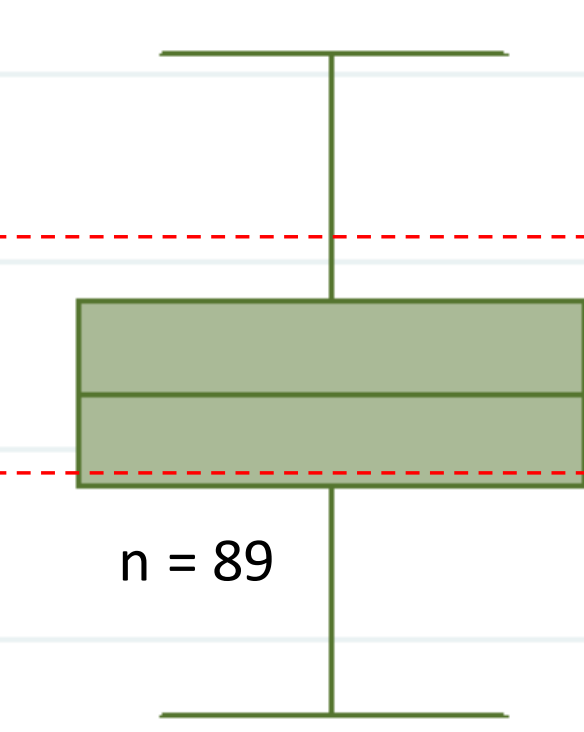

Post menopausal

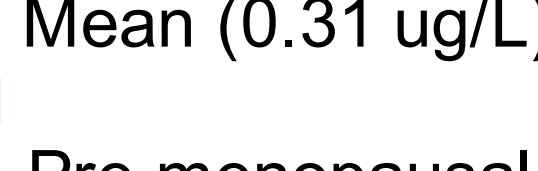

Pre menopausal

Figure 3: CTX at 0, 4, 12 months for defined populations a) $\leq$ pre-menopausal mean at baseline

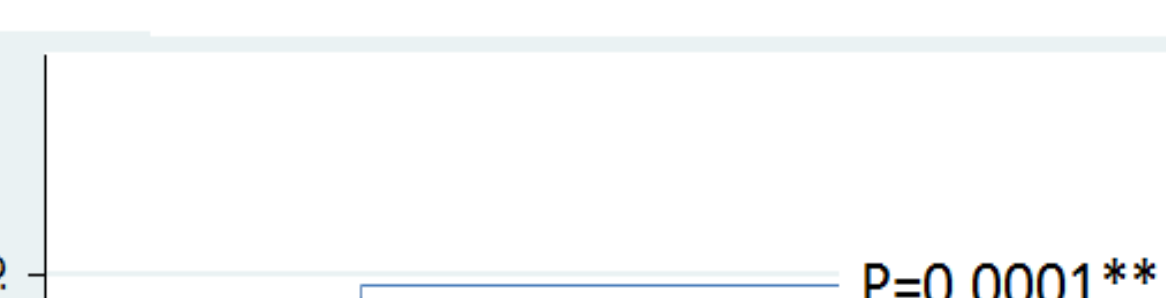

-
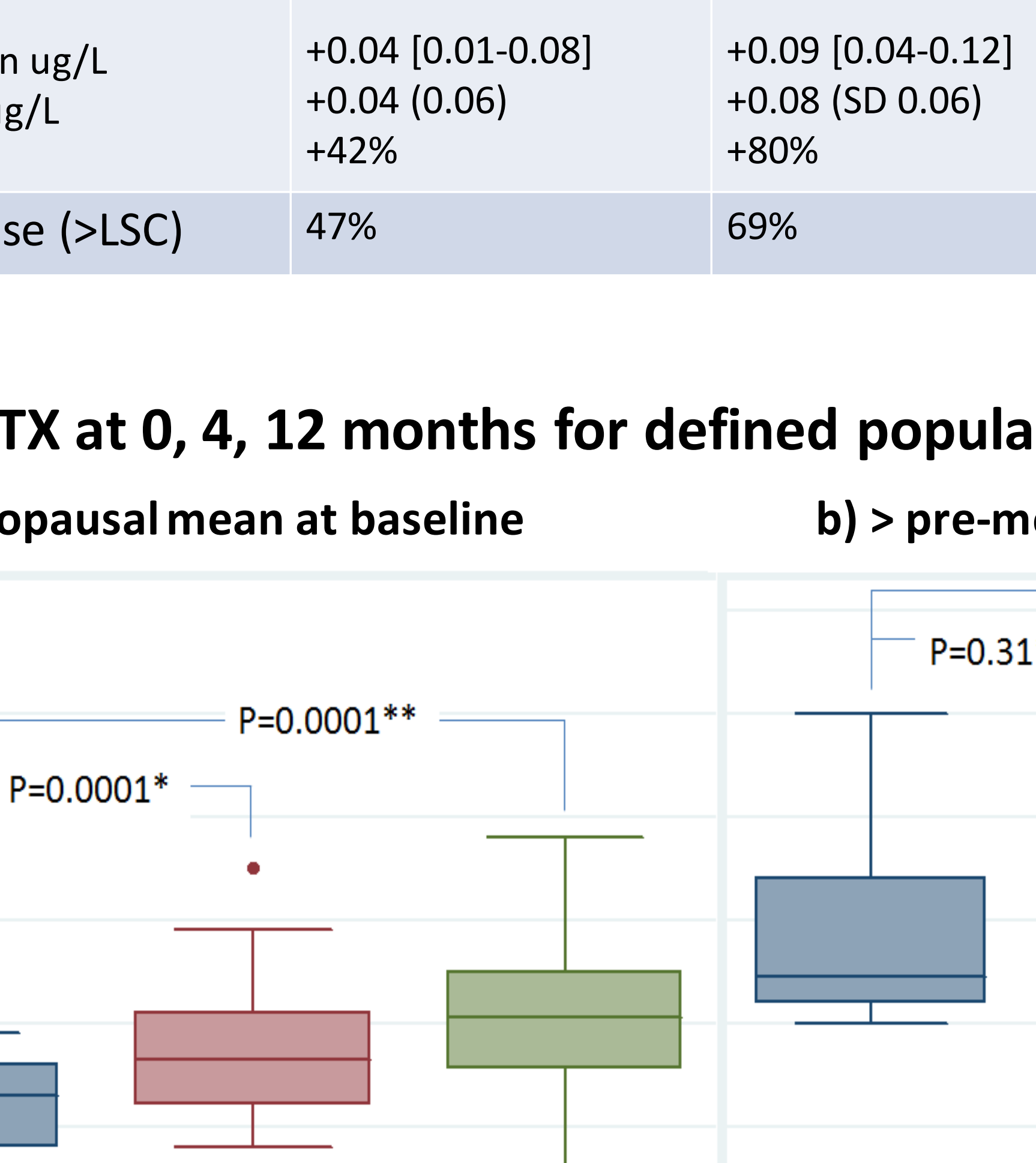

+0.08 (SD 0.06)

$69 \%$
Overall population (figure 2):

- Detectable rise in CTX seen from as early as 4 months in $47 \%$ patients; $69 \%$ at 12 months

\section{Subpopulations (figure 3):}

-If CTX $\leq$ pre-menopausal mean (i.e. treatment target) at baseline, statistically significant increases in CTX seen at 4 and 12 months

-If CTX > pre-menopausal mean at baseline, no significant change at 4 months, significant by 12 months

- No significant difference between Alendronic acid and Risedronate seen (data not shown)

Monitoring outcomes using population data (figure 4):

- Baseline CTX not suppressed to premenopausal mean level after 5 yrs of BP use in 32\% patients

-Where CTX suppressed at baseline:

At 4 months $28 \%$ had significant rise in CTX that was also above mean level (?consider re-start)

At 12 months this had risen to 53\%; $47 \%$ CTX still suppressed at this stage
Figure 4: CTX monitoring outcomes at baseline, 4 and 12 months

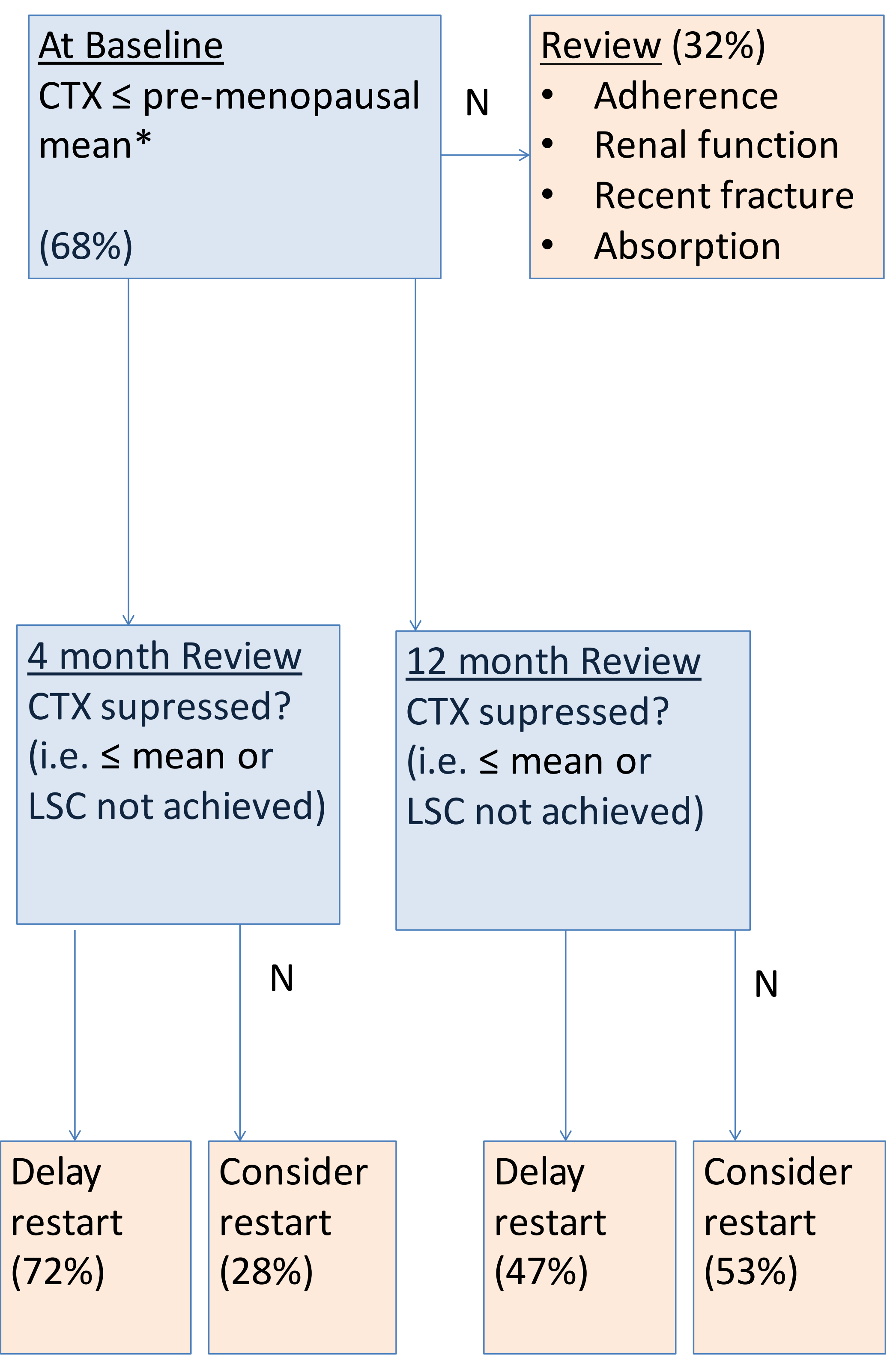

\section{Conclusion}

- After at least 5 years of treatment, CTX may not be adequately suppressed in a third of patients. Drug adherence and therapy choice should be reviewed in this group.

- Less significant changes in CTX seen if levels not adequately suppressed at baseline ?adherence

- Treatment effects can wear off as quickly as 4 months, but may also be maintained for 12 months

- Monitoring of CTX can potentially be used to identify these patients, some of whom may need to re-start treatment earlier

\section{References}

1.Sorensen $\mathrm{OH}$ et al (2003) Long-term efficacy of risedronate: a 5-year placebo-controlled clinical experience. Bone;32(2):120-6.; 2.Black DM et al (2006) Effects of continuing or stopping alendronate after 5 years of treatment: the Fracture Intervention Trial Long-term Extension (FLEX): a randomized trial. JAMA ; 296; 24;2927-2938;

3.MHRA Drug Safety Update June 2011, vol 4 issue 11: A1;

4. Gossiel F et al (2014) Establishing reference intervals for bone turnover markers in healthy postmenopausal women in a nonfasting state. BoneKEy Reports 3, article no: 573. 